Review Article

\title{
Structure-Function Relationship in Leishmanial Globin Coupled Soluble Adenylate Cyclase
}

\author{
SUMIT SEN SANTARA ${ }^{1, *}$ and SUBRATA ADAK ${ }^{2}$ \\ 'Program in Cellular and Molecular Medicine, Boston Children's Hospital and Department of Pediatrics, \\ Harvard Medical School, Boston MA 02115 USA \\ ${ }^{2}$ Structural Biology and Bio-Informatics Division, CSIR-Indian Institute of Chemical Biology, 4, Raja S. \\ C. Mullick Road, Kolkata 700 032, India
}

(Received on 01 August 2018; Accepted on 08 September 2019)

\begin{abstract}
Leishmania promastigotes inhabit the midgut of sandfly, where they are densely packed together and the environment of these promastigotes is then likely to become hypoxic or even anoxic. Interestingly, Leishmania can survive in low oxygen concentrations, which is an exception from Trypanosoma brucei. Although some transcriptional regulators in mammals help to respond in adaptive responses during hypoxia, Leishmania lacks hypoxic inducible factor (HIF) as well as transcriptional regulation. Thus, the underlying mechanism still remains unclear. Recently, our laboratory has discovered a globin coupled heme containing adenylate cyclase from $L$. major called HemAC-Lm, which is likely to function in cellular adaptability under various $\mathrm{O}_{2}$ limiting conditions. HemAC-Lm proteins usually consist of an $\mathrm{N}$-terminal heme-containing $\mathrm{O}_{2}$ sensor domain and a $\mathrm{C}$-terminal adenylate cyclase domain. The $\mathrm{N}$-terminal heme-containing $\mathrm{O}_{2}$ sensor domain has two globin domains (globin-A and globin-B). Biochemical studies of wild type and mutant proteins further suggest that the oxygenated form of heme is only present in globin-A domain, leading to maximum cAMP production. Furthermore, the data from HemAC-Lm knock down, as well as overexpressed cells, suggest that cAMP generation shows stimulatory, as well as inhibitory role in cell proliferation during normoxia. In addition, $\mathrm{O}_{2}$-dependent cAMP signaling via protein kinase A plays a fundamental role in cell survival through suppression of oxidative stress under hypoxia.
\end{abstract}

Keywords: Leishmania; Heme Protein; Hypoxia; Adenylate Cyclase; Oxygen Sensor Proteins

\section{Introduction}

Adaptation to a changing environment is the basic characteristic of all living organisms. Several sensor proteins thus have evolved with time to detect and respond to environmental changes. All organisms express heme sensor proteins to regulate small gaseous molecules like $\mathrm{O}_{2}, \mathrm{CO}$ and $\mathrm{NO}$ that regulate numerous important cellular pathways (Girvan and Munro, 2013). In the past few years, the heme-based sensor field bloomed with the discovery of several new heme-binding sensory domains including heme nitric oxide/oxygen (H-NOX) binding domains, heme PAS domains, CooA proteins, heme-binding SCHIC domains, heme GAF domains, and sensor globin domains. Generally, heme based sensors consist of a sensor domains that link to the transmitter domains with variety of biological functions like histidine kinases, phosphodiesterases, DNA-binding domains, guanylate cyclases, diguanylate cyclase, and methyl accepting chemotaxis protein (Farhana et al., 2012). Globin-coupled sensor (GCS) proteins were first discovered in bacterial aerotaxis before the discovery of globin-coupled heme containing adenylate cyclase (HemAC-Lm) from the unicellular eukaryotic organism Leishmania, GCSs are known to exist only in the prokaryotic world.

Adenosine 32, 52-cyclic monophosphate (cAMP) is a vital signaling molecule that acts as a key second messenger in diverse biological functions, including proliferation, survival, differentiation,

*Author for Correspondence: E-mail: sumit.santara@childrens.harvard.edu 
migration and programmed cell death. cAMP is universally generated by adenylate cyclase (AC), which catalyzes the cyclization of ATP to cAMP. AC is regulated by various molecules including bicarbonate, calcium, and hormones (Hanoune and Defer, 2001; Steegborn, 2014). Several heme based oxygen sensor proteins are known to be associated with cyclic nucleotide synthesis, including heme containing diguanylate cyclase (Hem-DGC), heme based YddV and BPeGreg (globin coupled diguanylate cyclase from Bordetella pertusis). HemAC-Lm is the only characterized heme containing oxygen regulated AC. However, the cAMP signaling pathways in protozoan parasites, belonging to the order Kinetoplastida differ a lot from their mammalian hosts. Genomic analyses suggest that these parasites lack heterotrimeric $\mathrm{G}$ protein, conventional transcription factors, as well as classical cAMP effectors, such as cAMP gated channels or cAMP secretory channels, although they do have the receptor-type ACs that are topologically similar to GC-coupled receptors of higher eukaryotes. Both ACs belong to a family of class III $\mathrm{AC}$ which are widely distributed from unicellular to multicellular organisms, forming the biggest family of ACs (Salmon et al., 2012). Throughout this minireview, the globin-coupled heme containing adenylate cyclase from Leishmania major will be presented from a structure-function point of view, emphasizing its contribution to the parasite survival under limited oxygen conditions.

\section{Primary Structure of HemAC-Lm}

HemAC-Lm is 616 amino acids long and is composed of $\mathrm{N}$ terminal globin-A (78-209 amino acid) domain, followed by globin-B (210-360 amino acid) domain which is linked with $C$ terminal adenylate cyclase (361616) domain (Figure 1A). Both globinA and globinB domains share sequence and structural homology with other globin domains, including mammalian myoglobin, cytoglobin and neuroglobin. However, when compared to hemoglobin and myoglobin they lack the D helix and have a shortened E helix (Sen Santara et al., 2013). The absolutely conserved residues in all globins are the proximal His in the F helix, Pro in C helix, and Phe in the CD1 region (J Mol Biol 196(1):199-216). All three residues, Pro115, Phe121, and His161, are conserved in this enzyme. A key feature of Globin-A is its six-coordinate heme structure in the ferrous states, with the proximal histidine (His161) and an endogenous unknown distal residue (Roy et al., 2014). Furthermore, SWISS-MODEL protein modeling also predicts His 311 that acts as the proximal ironcoordinating ligand in globin B but lacks the distal His residue which is crucial for stabilizing ferrous- $\mathrm{O}_{2}$ state. Globin $\mathrm{B}$ domain is linked to the $\mathrm{C}$ terminus $\mathrm{AC}$ domain, which contains characteristic lysine (Lys-427) and aspartic acid (Asp-508) as conserved residues for catalysis (Roy et al., 2015).

\section{Ligand Binding Characteristic and Mechanism of Catalysis}

Biochemical and spectroscopic characterization of full length wild type, truncated and site-specific mutant proteins have provided the role of globin-A connecting globin-B domain in catalysis. This study also characterizes the specific amino acid(s) or region(s) of HemAC-lm responsible for heme binding, and the mechanism by which the heme and connecting globin$\mathrm{B}$ domain regulates $\mathrm{AC}$ activity. Characteristic absorption spectra of full length HemAC-Lm is similar to oxygen-bound heme proteins with absorption maxima of 414 (Soret), 575 (á-band), and $538 \mathrm{~nm}$ ( $\beta$ band). Native full length protein binds with $\mathrm{CO}$, and new peaks appear at 419 (Soret), 573 ( $\alpha$-band), and $535 \mathrm{~nm}$ ( $\alpha$-band), suggesting that the native form of HemAC-Lm is in the ferrous state. Upon addition of dithionite into the native protein, the oxygenated full length form of the HemAC-Lm protein shifted the Soret band from 414 to $423 \mathrm{~nm}$, and simultaneously, the $\alpha \beta$-band appeared at 560 and $532 \mathrm{~nm}$. This spectral characteristic is similar to deoxyneuroglobin/ deoxycytoglobin, indicating the formation of ferrous six-coordinate low-spin heme state. Although the oxygen bound absorption spectra of both full length HemAC-Lm and globin-A domain deleted proteins $(\Delta 209)$ showed similar low-spin heme state, but the deoxy $\Delta 209$ protein displayed the Soret band at 428 $\mathrm{nm}$ and the $\alpha \beta$-band at $556 \mathrm{~nm}$ (like deoxyhemoglobin/ deoxymyoglobin), indicating that the globin-A domain deleted proteins show ferrous five-coordinate highspin heme state. $\Delta 209$ proteins bind with $C O$, indicating that the native form of globin $\mathrm{B}$ is in the ferrous- $\mathrm{O}_{2}$ state (Roy et al., 2015; Sen Santara et al. 2013). Heme content studies of the H161A (heme binding residue of GlobinA domain) and $\mathrm{H} 311 \mathrm{~A}$ (heme binding residue of GlobinB domain) mutants in full length protein showed that only His 161 residue is responsible for heme binding (Roy et al., 2015). 
The size exclusion chromatography of purified full-length protein and localization studies suggest that HemAC-Lm forms a dimer and localize in the cytosol of Leishmania major promastigotes (Sen Santara et al., 2013). HemAC-Lm catalyzed only the conversion of ATP to cAMP not GTP to cGMP, suggesting that the enzyme utilizes only ATP as substrate. The adenylate cyclase activity of HemAC-Lm is induced by $\mathrm{O}_{2}$ or $\mathrm{CO}$-binding indicating that activation is due to binding of the molecule with heme. $\mathrm{O}_{2}$-dependent spectral shift of deoxy enzyme and $\mathrm{AC}$ activity by sequential addition of $\mathrm{O}_{2}$ demonstrate that adenylate cyclase activity is directly proportional to the conversion of the oxy form of full length enzyme. ATP dependent initial velocity of reactions was fitted into the Michaelis-Menten equation with the $k_{\text {cat }}$ value $27 \pm 1.1 \mathrm{mim}^{-1}$ and $\mathrm{K}_{\mathrm{m}}$ value $\sim 1.9 \mathrm{mM}$ (SenSantara et al., 2013). The rate of cAMP synthesis for full length ferric, apo (heme free) forms and truncated catalytic domain $(\Delta 360)$ are 10 -fold lower than wild type enzyme, whereas the catalytic activities of $\Delta 209$ and H311A- $\Delta 209$ (apo protein) have no detectable activities (Roy et al., 2015; Roy et al., 2014). Comparative activity studies among full length wild type, $\Delta 209$ and $\Delta 360$ proteins suggest that the globin$\mathrm{B}$ domain inhibits catalytic activity of the $\mathrm{AC}$ domain, whereas globin-A domain may change the protein conformation of the HemAC-Lm and relieve the globin-B domain induced suppression. Interestingly, these findings were validated by in vivo complementation experiments in E. coli TP610 (Roy et al., 2015), which carries a defect in the adenylyl cyclase gene. As only complemented strain can produce active $\beta$-galactosidase enzymes, it forms blue colonies on X-gal plates (Taylor et al., 1999).

How the suppressive interactions between globin B and adenylate cyclase domain are relieved in a single subunit can be best explained by the domain swapping model shown in Fig. 1B. According to this

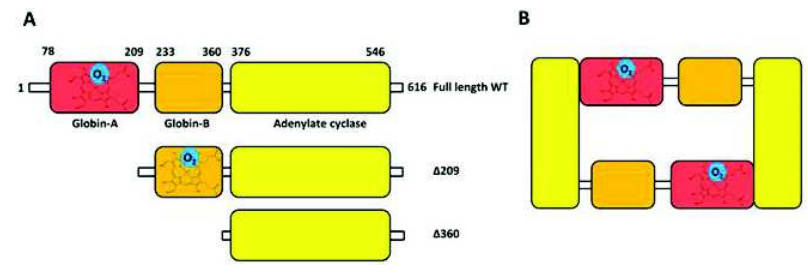

Fig. 1: Schematic of domain architecture of HemAC-Lm (A) Proposed model for active dimeric form of HemACLm (B) model, $\mathrm{O}_{2}$-dependent conformational changes in the globinA domain that sequentially stimulate catalytic activity of the AC domain located on adjacent subunits. This arrangement may circumvent a physical barrier for interaction between globin B domain and the AC domain located on same subunit. Domain swapping is a comparatively common characteristic among heme proteins, like cytochrome c, myoglobin (Lin et al., 2015), NO synthase (Siddhanta et al., 1998), nitrite reductase (Heiss et al., 1995), hemophore HasA (Czjzek et al., 2007) and methyl accepting chemotaxis protein (Silva et al., 2012), which allows to adopt different functional state of the protein. In evolutionary context, the inter-subunit interactions are preferred over, interaction between domains (intrasubunit) of a multidomain protein, because the intra-subunit interaction does not create any selective pressure to be preserved after oligomerization (Bennett et al., 1995). Thus, the multi domain structure of HemACLm probably evolved first, followed by its inter-subunit interactions between adjacent $\mathrm{AC}$ and globin-A domains for regulation.

\section{HemAC-Lm mediated Signalling in Leishmania}

cAMP signaling has been implicated as one of the major environmental sensing machineries in many unicellular eukaryotes like Trypanosoma, Toxoplasma, Plasmodium and others (Tagoe et al., 2015). Overexpression and knock down of hemecontaining AC (HemAC-Lm) in Leishmania major promastigotes under normoxic conditions, lead to alterations in cell shape and slower growth rate without compromising cell survival. These data strongly suggest that the typical level of cAMP plays a positive role, whereas an excess or shortage of cAMP plays a negative role in the initiation of cell proliferation under normoxia (Pawelek et al., 1975). However, under hypoxic condition HemAC-Lm overexpression and knock down cells are less viable than control cells. These results suggest that HemAC-Lm is an essential gene but excessive cAMP is toxic to the cell (Bronstad et al., 1983). For validation of the above results, we found similar percentage of cell death in the presence or absence of different concentrations of CPT-cAMP and PKA inhibitors (H89 and PKI). The results of PKA inhibitor suggest that cAMP signaling occurred through protein kinase $\mathrm{A}$. It is also shown that $\mathrm{O}_{2}{ }^{-}$ dependent cAMP generation by HemAC-Lm is a key factor that maintains the optimum level of anti-oxidant 
enzymes, including glutathione peroxidase, peroxidoxin and superoxide dismutase through the activation of PKA (Sen Santara et al., 2013). However, it is really unknown what triggers the expression of these antioxidant genes in Leishmania because the parasite is lacking transcriptional regulation. Studies from another group showed that ACs from kinetoplastida acts as an environmental sensors and controllers of host innate immune response (Saada et al., 2014; Salmon et al., 2012). Parasites that are pre-exposed to environmental stress $(\mathrm{pH} 5.5$ and temperature $37^{\circ} \mathrm{C}$ ) have been shown to induce resistance against oxidative damage (Miller et al., 2000; Zarley et al., 1991). Earlier results also showed that disruption of anti-oxidant genes in parasites make them more susceptible to ROS inducing agents or intracellular killing in the macrophages (Barr and Gedamu, 2001; Ghosh et al., 2003; Tovar et al., 1998). Overexpressed and knock down cells show more pronounced oxidative stress compared to control parasite during hypoxia indicating that excess or shortage of cAMP produced excessive ROS, which is toxic to the parasite at limited oxygen levels. The attenuation of ROS by using N-acetyl-L-cysteine (antioxidant) abolished the acceleration of cell death seen during hypoxia, indicating that $\mathrm{O}_{2}$-dependent cAMP regulation in control cells is required for the protection of cells against the accelerated rates of cell death. Altogether, the schematic diagram (Fig. 2) shows a possible role of leishmanial HemAC in the adaptation of parasites at various concentrations of environmental oxygen.

\section{Concluding Remarks and Perspectives}

Like in other Trypanosomes, leishmanial receptor adenylatecyclases (LdRACs) are known for their differential expression, localization and diversity of function. HemAC-Lm which is the most recently discovered source of cAMP in Leishmania parasite, acts as a sensor for the external gaseous ligand like oxygen. HemAC-Lm is found diffusely distributed in the cytoplasm of promastigotes, although it's localization in amastigotes or during hypoxia is not yet known. The mammalian cytoplasmic sAC translocates to the mitochondria during acidosis/ ischemia to promote the mitochondrial apoptotic pathway (Acin-Perez et al., 2009). The unique structural properties and biochemical characteristics of HemAC-Lm makes it an ideal target for therapeutic approaches. HemAC-Lm is distantly related to the

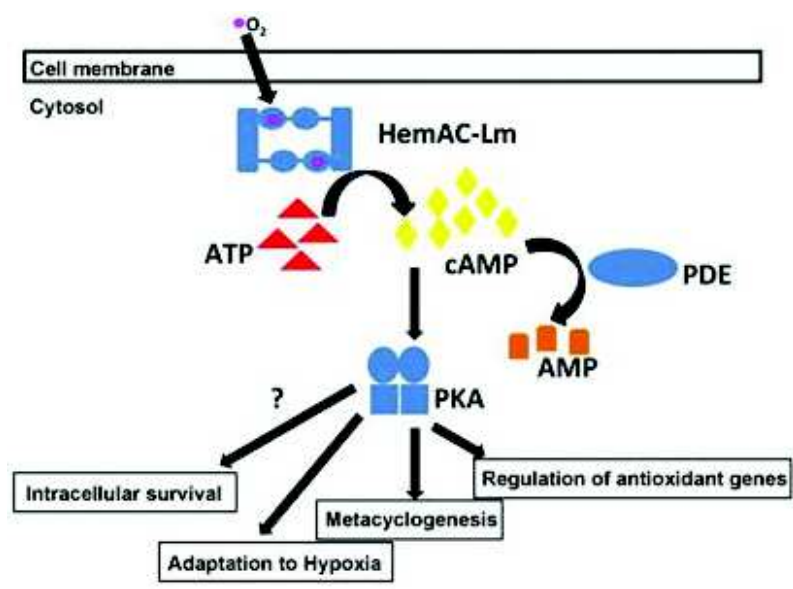

Fig. 2: Schematic representation of cAMP signaling via HemAC-Im in Leishmania parasite. HemAC-Lm primarily localized in the cytosol of Leishmania and catalyses cAMP from ATP when it binds to oxygen. cAMP activates PKA enzyme which leads to the optimal expression of antioxidant genes like peroxidoxin, superoxide dismutase and tryparedoxin peroxidase, differentiation in infective metacyclic stage of the parasite, increased survival during hypoxia. HemACLm generated cAMP may also have implication in intracellular survival in mammalian host. Intracellular cAMP pool is regulated by a stage specific phosphodiesterase enzymes that converts cAMP to AMP

mammalian sAC and it is also found to be insensitive to the mammalian sAC inhibitor KH7. Different component of the cAMP pathway (like phosphodiesterase) of Leishmania have already been tested for safe and effective anti-leishmanial drug target with little success. Therefore, HemAC-Lm provides an alternative drug target for effective antileishmanial therapy. Mammalian tmACs, as well as, $\mathrm{sAC}$ are thought to function through a signaling microdomain that comprises A-kinase anchoring proteins (AKAPs), which tether PKA and PDEs to regulate cAMP diffusion or cross-communication. Several AKAP-like proteins are reported in unicellular parasites like PfAKAL from $P$. falciparum merozoites and gametocytes (Bandje et al., 2016). Although HemAC-Lm is known to activate PKA, involvement of any putative AKAP is not characterized yet. Several studies on different human cell lines showed direct involvement of mitochondrial oxidative phosphorylation (OXPHOS) complexes in hypoxia induced ROS production that lead to the activation of the hypoxia-inducible factor (HIF) 
pathway which ultimately modulates gene expression (Movafagh et al., 2015). Very little is known about the role of cAMP in hypoxia as well as the exact function of neuroglobin and cytoglobin in oxygen homeostasis and hypoxia protection. HemAC-Lm might provide an important clue for the unknown

\section{References}

Acin-Perez R, Salazar E, Kamenetsky M, Buck J, Levin L R, et al. (2009) Cyclic AMP produced inside mitochondria regulates oxidative phosphorylation Cell Metab 9 265276

Bandje K, Naissant B, Bigey P, Lohezic M, Vayssieres M, et al. (2016) Characterization of an A-kinase anchoring proteinlike suggests an alternative way of PKA anchoring in Plasmodium falciparum Malar J 15248

Barr S D and Gedamu L (2001) Cloning and characterization of three differentially expressed peroxidoxin genes from Leishmania chagasi. Evidence for an enzymatic detoxification of hydroxyl radicals J Biol Chem 27634279 34287

Bennett M J, Schlunegger M P and Eisenberg D (1995) 3D domain swapping: a mechanism for oligomer assembly Protein $\mathrm{Sci}$ $42455-2468$

Bronstad G O, Sand T E and Christoffersen T (1983) Bidirectional concentration-dependent effects of glucagon and dibutyryl cyclic AMP on DNA synthesis in cultured adult rat hepatocytes Biochim Biophys Acta 763 58-63

Czjzek M, Letoffe S, Wandersman C, Delepierre M, Lecroisey A, et al. (2007) The crystal structure of the secreted dimeric form of the hemophore HasA reveals a domain swapping with an exchanged heme ligand $J$ Mol Biol 365 1176-1186

Farhana A, Saini V, Kumar A, Lancaster J R, Jr., Steyn A J (2012) Environmental heme-based sensor proteins: Implications for understanding bacterial pathogenesis Antioxid Redox Signal 17 1232-1245

Ghosh S, Goswami S and Adhya S (2003) Role of superoxide dismutase in survival of Leishmania within the macrophage Biochem J 369 447-452

Girvan H M and Munro A W (2013) Heme sensor proteins J Biol Chem 288 13194-13203

Hanoune J and Defer N (2001) Regulation and role of adenylyl cyclase isoforms Annu Rev Pharmacol Toxicol 41 145174

Heiss K F, Clark R H, Cornish J D, Stovroff M, Ricketts R, et al. (1995) Preferential use of venovenous extracorporeal function of neuroglobin and cytoglobin which could interact with different $\mathrm{sAC}$ or tmAC that lead to $\mathrm{O}_{2}$ regulated cAMP production. Further structure function and cell biological studies are needed to validate HemAC-Lm's candidature as an anti leishmanial drug target as well as its implication in human biology.

membrane oxygenation for congenital diaphragmatic hernia J Pediatr Surg 30 416-419

Lin Y W, Nagao S, Zhang M, Shomura Y, Higuchi Y, et al. (2015) Rational design of heterodimeric protein using domain swapping for myoglobin Angew Chem Int Ed Engl 54 511515

Miller M A, McGowan S E, Gantt K R, Champion M, Novick S $\mathrm{L}$, et al. (2000) Inducible resistance to oxidant stress in the protozoan Leishmania chagasi J Biol Chem 275 3388333889

Movafagh S, Crook S and Vo K (2015) Regulation of hypoxiainducible factor-1a by reactive oxygen species: new developments in an old debate $J$ Cell Biochem 116 696703

Pawelek J, Halaban R and Christie G (1975) Melanoma cells which require cyclic AMP for growth Nature 258 539-540

Roy J, Sen Santara S, Adhikari A, Mukherjee A and Adak S (2015) Control of catalysis in globin coupled adenylate cyclase by a globin-B domain Arch Biochem Biophys 579 $85-90$

Roy J, Sen Santara S, Bose M, Mukherjee S, Saha R, et al. (2014) The ferrous-dioxy complex of Leishmania major globin coupled heme containing adenylate cyclase: The role of proximal histidine on its stability Biochim Biophys Acta 1844 615-622

Saada E A, Kabututu Z P, Lopez M, Shimogawa M M, Langousis $\mathrm{G}$, et al. (2014) Insect stage-specific receptor adenylate cyclases are localized to distinct subdomains of the Trypanosoma brucei Flagellar membrane Eukaryot Cell 13 1064-1076

Salmon D, Vanwalleghem G, Morias Y, Denoeud J, Krumbholz C, et al. (2012) Adenylate cyclases of Trypanosoma brucei inhibit the innate immune response of the host Science 337 463-466

Sen Santara S, Roy J, Mukherjee S, Bose M, Saha R, et al. (2013) Globin-coupled heme containing oxygen sensor soluble adenylate cyclase in Leishmania prevents cell death during hypoxia Proc Natl Acad Sci U S A 110 16790-16795

Siddhanta U, Presta A, Fan B, Wolan D, Rousseau D L, et al. 
(1998) Domain swapping in inducible nitric-oxide synthase.

Electron transfer occurs between flavin and heme groups located on adjacent subunits in the dimer J Biol Chem $\mathbf{2 7 3}$ 18950-18958

Silva M A, Lucas T G, Salgueiro C A and Gomes C M (2012) Protein folding modulates the swapped dimerization mechanism of methyl-accepting chemotaxis heme sensors PLoS One 7 e 46328

Steegborn C (2014) Structure, mechanism, and regulation of soluble adenylyl cyclases - similarities and differences to transmembrane adenylyl cyclases Biochim Biophys Acta 1842 2535-2547

Tagoe D N, Kalejaiye T D and de Koning H P (2015) The ever unfolding story of cAMP signaling in trypanosomatids: Vive la difference! Front Pharmacol 6185
Taylor M C, Muhia D K, Baker D A, Mondragon A, Schaap P B, et al. (1999) Trypanosoma cruzi adenylyl cyclase is encoded by a complex multigene family Mol Biochem Parasitol 104 205-217

Tovar J, Cunningham M L, Smith A C, Croft S L and Fairlamb A H (1998) Down-regulation of Leishmania donovani trypanothione reductase by heterologous expression of a trans-dominant mutant homologue: Effect on parasite intracellular survival Proc Natl Acad Sci U S A 955311 5316

Zarley J H, Britigan B E and Wilson M E (1991) Hydrogen peroxide-mediated toxicity for Leishmania donovani chagasi promastigotes. Role of hydroxyl radical and protection by heat shock J Clin Invest 88 1511-1521. 